\title{
Effects of Hydrodynamic Interactions on the Near-Surface Diffusion of Spheroidal Molecules
}

Paweł Czajka ${ }^{1}$, Jan M. Antosiewicz ${ }^{2}$ and Maciej Długosz ${ }^{2, *}$

${ }^{1}$ Faculty of Physics, University of Warsaw, 02-105 Warsaw, Pasteura 5, Poland

${ }^{2}$ Division of Biophysics, Institute of Experimental Physics, Faculty of Physics, University of Warsaw, 02-105 Warsaw, Pasteura 5, Poland

${ }^{*}$ Corresponding author: Maciej Długosz, email: Maciej.Dlugosz@fuw.edu.pl, phone: +482255 32229 


\section{Near-Surface Resistance Tensor of a Prolate Ellipsoid of Revolution}

The full set of expressions for components of the near-wall correction for the bulk resistance tensor of an axisymmetric particle (in our case a prolate spheroid or a prolate ellipsoid of revolution) derived by Lisicki and co-authors [1] is given below.

Coordinate frame used in [1] to evaluate the correction is presented in Figure S1. The laboratory frame of reference consists of three basis vectors, pointing in Cartesian directions $x, y$, $z$ (the basis set consists of three unit vectors $\left\{\hat{e}_{x}, \hat{e}_{y}, \hat{e}_{z}\right\}$ ), with the $z$ axis normal to the surface of the wall. The body-wall frame consists of three basis vectors, $\left\{\hat{u}, \hat{u}_{\perp 1}, \hat{u}_{\perp 2}\right\}$, with $\hat{u}$ pointing along the long axis of the particle, $\hat{u}_{\perp 1}=\left(\hat{e}_{z} \times \hat{u}\right) /\left|\hat{e}_{z} \times \hat{u}\right|$, and $\hat{u}_{\perp 2}=\hat{u}_{\perp 1} \times \hat{u}$. In the body-wall frame, the resistance tensor depends on the elevation of the body above the plane $(z)$ and the inclination angle $\theta$, with $\cos \theta=\hat{e}_{z} \cdot \hat{u}$.

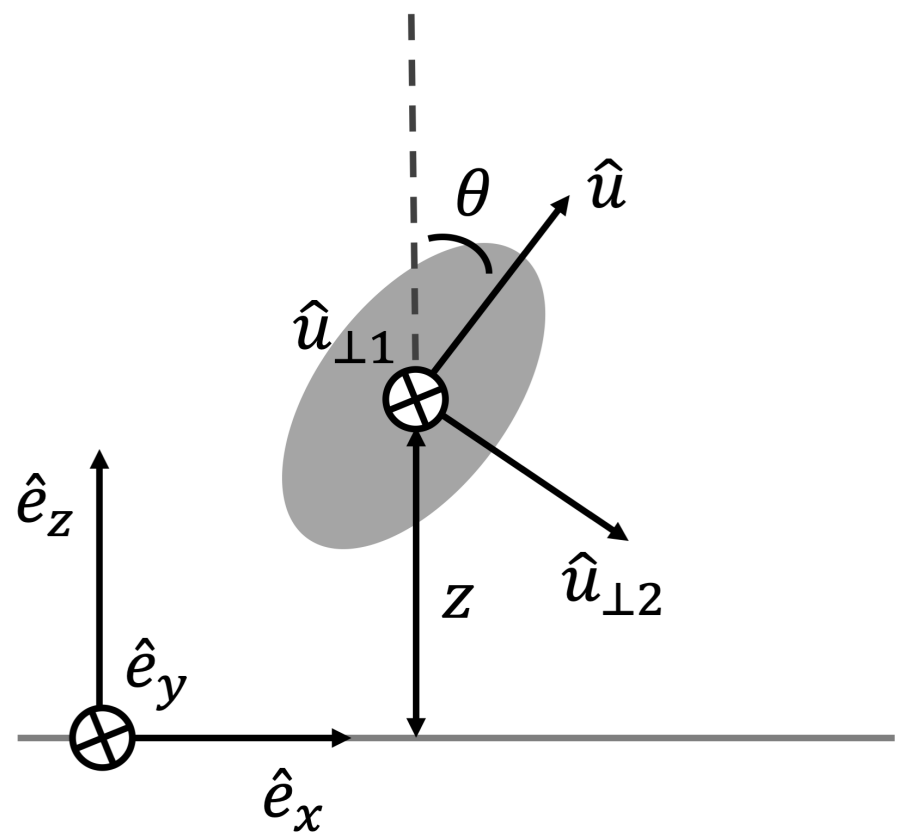

Figure S1: Schematic representation of a prolate ellipsoid near the planar surface and the body-wall coordinate system employed in [1].

Resistance tensor matrix in the body-wall frame $\left\{\hat{u}, \hat{u}_{\perp 1}, \hat{u}_{\perp 2}\right\}$ is constructed as a sum of the resistance tensor matrix $\boldsymbol{\Xi}_{o}$ of the body suspended in an unbounded fluid and the correcting matrix $\Delta \boldsymbol{\Xi}$, whose components are calculated for a given $z$ and $\theta$ :

$$
\boldsymbol{\Xi}=\boldsymbol{\Xi}_{o}+\Delta \boldsymbol{\Xi}=\left(\begin{array}{cc}
\boldsymbol{\Xi}_{o}^{T T} & \boldsymbol{\Xi}_{o}^{T R} \\
\boldsymbol{\Xi}_{o}^{R T} & \boldsymbol{\Xi}_{o}^{R R}
\end{array}\right)+\left(\begin{array}{cc}
\Delta \boldsymbol{\Xi}^{T T} & \Delta \boldsymbol{\Xi}^{T R} \\
\Delta \boldsymbol{\Xi}^{R T} & \Delta \boldsymbol{\Xi}^{R R}
\end{array}\right)
$$

In the body-wall frame, the bulk resistance tensor, $\boldsymbol{\Xi}_{o}$, is independent of the position and orientation of the ellipsoid relative to the wall. Its matrix is diagonal: 


$$
\Xi_{o}=\left(\begin{array}{cccccc}
\Xi_{\|}^{T T} & 0 & 0 & 0 & 0 & 0 \\
0 & \Xi_{\perp}^{T T} & 0 & 0 & 0 & 0 \\
0 & 0 & \Xi_{\perp}^{T T} & 0 & 0 & 0 \\
0 & 0 & 0 & \Xi_{\|}^{R R} & 0 & 0 \\
0 & 0 & 0 & 0 & \Xi_{\perp}^{R R} & 0 \\
0 & 0 & 0 & 0 & 0 & \Xi_{\perp}^{R R}
\end{array}\right)
$$

and its components are defined as [2]:

$$
\begin{gathered}
\Xi_{\|}^{T T}=(6 \pi \eta a) \frac{8}{3} \varepsilon^{3}\left(-2 \varepsilon+\left(1+\varepsilon^{2}\right) \ln \left(\frac{1+\varepsilon}{1-\varepsilon}\right)\right)^{-1} \\
\Xi_{\perp}^{T T}=(6 \pi \eta a) \frac{16}{3} \varepsilon^{3}\left(2 \varepsilon+\left(3 \varepsilon^{2}-1\right) \ln \left(\frac{1+\varepsilon}{1-\varepsilon}\right)\right)^{-1} \\
\Xi_{\|}^{R R}=\left(8 \pi \eta a^{3}\right) \frac{4}{3} \varepsilon^{3}\left(1-\varepsilon^{2}\right)\left(2 \varepsilon-\left(1-\varepsilon^{2}\right) \ln \left(\frac{1+\varepsilon}{1-\varepsilon}\right)\right)^{-1} \\
\Xi_{\perp}^{R R}=\left(8 \pi \eta a^{3}\right) \frac{4}{3} \varepsilon^{3}\left(2-\varepsilon^{2}\right)\left(-2 \varepsilon+\left(1+\varepsilon^{2}\right) \ln \left(\frac{1+\varepsilon}{1-\varepsilon}\right)\right)^{-1} \\
\varepsilon=\sqrt{1-\frac{b^{2}}{a^{2}}}
\end{gathered}
$$

Components of the correction matrix, $\Delta \boldsymbol{\Xi}$ can be calculated as follows [1]:

$$
\begin{aligned}
& \Delta \boldsymbol{\Xi}^{T T}=-\frac{1}{16 \pi \eta H} \boldsymbol{A}_{1}+\frac{1}{(16 \pi \eta z)^{2}} \boldsymbol{A}_{2} \\
& \boldsymbol{\Delta} \boldsymbol{\Xi}^{T R}=-\frac{1}{32 \pi \eta z^{2}} \boldsymbol{B} \\
& \Delta \boldsymbol{\Xi}^{R T}=-\frac{1}{32 \pi \eta z^{2}} \boldsymbol{B}^{T} \\
& \Delta \boldsymbol{\Xi}^{R R}=-\frac{1}{64 \pi \eta z^{3}} \boldsymbol{C} \\
& \boldsymbol{A}_{1}=-\frac{3}{2}\left(\begin{array}{ccc}
\left(\Xi_{\|}^{T T}\right)^{2}\left(1+\cos ^{2} \theta\right) & 0 & -\Xi_{\|}^{T T} \Xi_{\perp}^{T T} \sin \theta \cos \theta \\
0 & \left(\Xi_{\perp}^{T T}\right)^{2} & 0 \\
-\Xi_{\|}^{T T} \Xi_{\perp}^{T T} \sin \theta \cos \theta & 0 & \left(\Xi_{\perp}^{T T}\right)^{2}\left(1+\sin ^{2} \theta\right)
\end{array}\right) \\
& \boldsymbol{A}_{2}=\frac{9}{4}\left(\begin{array}{ccc}
A_{\|} & 0 & A_{\| \perp} \\
0 & A_{\perp 1} & 0 \\
A_{\| \perp} & 0 & A_{\perp 2}
\end{array}\right) \\
& A_{\perp 1}=\left(\Xi_{\perp}^{T T}\right)^{3} \\
& A_{\|}=\left(\Xi_{\|}^{T T}\right)^{3}\left(1+\cos ^{2} \theta\right)^{2}+\Xi_{\perp}^{T T}\left(\Xi_{\|}^{T T}\right)^{2} \sin ^{2} \theta \cos ^{2} \theta \\
& A_{\perp 2}=\left(\Xi_{\perp}^{T T}\right)^{3}\left(1+\sin ^{2} \theta\right)^{2}+\left(\Xi_{\perp}^{T T}\right)^{2} \Xi_{\|}^{T T} \sin ^{2} \theta \cos ^{2} \theta \\
& A_{\| \perp}=-\Xi_{\|}^{T T} \Xi_{\perp}^{T T}\left(\Xi_{\|}^{T T}\left(1+\cos ^{2} \theta\right)+\Xi_{\perp}^{T T}\left(1+\sin ^{2} \theta\right)\right) \sin \theta \cos \theta
\end{aligned}
$$




$$
\begin{aligned}
& \boldsymbol{B}=\frac{3}{2}\left(4 \pi \eta a^{3} \xi^{D R}\right)\left(\begin{array}{ccc}
0 & \Xi_{\|}^{T T}\left(1+\cos ^{2} \theta\right) \sin \theta & 0 \\
0 & 0 & \Xi_{\perp}^{T T} \cos \theta \\
0 & -\Xi_{\perp}^{T T}\left(1+\sin ^{2} \theta\right) \cos \theta & 0
\end{array}\right) \\
& \boldsymbol{C}=-\frac{1}{2}\left(\begin{array}{ccc}
\left(\Xi_{\|}^{R R}\right)^{2}\left(5-3 \cos ^{2} \theta\right) & 0 & 3 \Xi_{\|}^{R R} \Xi_{\perp}^{R R} \sin \theta \cos \theta \\
0 & 5\left(\Xi_{\perp}^{R R}\right)^{2} & 0 \\
3 \Xi_{\|}^{R R} \Xi_{\perp}^{R R} \sin \theta \cos \theta & 0 & \left(\Xi_{\perp}^{R R}\right)^{2}\left(5-3 \sin ^{2} \theta\right)
\end{array}\right) \\
& +\frac{3}{2}\left(4 \pi \eta a^{3} \xi^{D R}\right)\left(\begin{array}{ccc}
0 & 0 & \Xi_{\|}^{R R} \sin \theta \cos \theta \\
0 & -2 \Xi_{\perp}^{R R}\left(1-2 \cos ^{2} \theta\right) & 0 \\
\Xi_{\|}^{R R} \sin \theta \cos \theta & 0 & 2 \Xi_{\perp}^{R R} \cos ^{2} \theta
\end{array}\right) \\
& -\frac{3}{2}\left(4 \pi \eta a^{3} \xi^{D R}\right)^{2}\left(\begin{array}{ccc}
0 & 0 & 0 \\
0 & 3+\cos ^{2} \theta-\cos ^{4} \theta & 0 \\
0 & 0 & 1+2 \cos ^{2} \theta
\end{array}\right)
\end{aligned}
$$

Where the $\xi^{D R}$ coefficient for a prolate ellipsoid of revolution is calculated as: [2]

$$
\xi^{D R}=\frac{8}{3} \varepsilon^{5}\left(-2 \varepsilon+\left(1+\varepsilon^{2}\right) \ln \left(\frac{1+\varepsilon}{1-\varepsilon}\right)\right)^{-1}
$$

Transformation of $\boldsymbol{\Xi}$ from the left-handed coordinate system $\left\{\hat{u}, \hat{u}_{\perp 1}, \hat{u}_{\perp 2}\right\}$ (see Figure S1) to the right-handed coordinate frame can be done via operation $\boldsymbol{U} \boldsymbol{\Xi} \boldsymbol{U}^{T}$, where the $\boldsymbol{U}$ matrix is defined as:

$$
\boldsymbol{U}=\left(\begin{array}{cccccc}
1 & 0 & 0 & 0 & 0 & 0 \\
0 & 1 & 0 & 0 & 0 & 0 \\
0 & 0 & -1 & 0 & 0 & 0 \\
0 & 0 & 0 & 1 & 0 & 0 \\
0 & 0 & 0 & 0 & 1 & 0 \\
0 & 0 & 0 & 0 & 0 & -1
\end{array}\right)
$$

After this operation, and after a rearrangement of rows and columns of the resulting matrix, we obtain the resistance tensor in the $\left\{\hat{v}_{1}, \hat{v}_{2}, \hat{v}_{3}\right\}$ frame employed in the current work.

\section{Brownian Dynamics Algorithm Verification}

In Figure S2 we compare components of the mobility tensor of a prolate ellipsoid (see equations 12 - 14), calculated either from Brownian dynamics simulations or based on analytical expressions [1] as described above, for a fixed elevation of the ellipsoid above the plane and for different values of the inclination angle $\theta$ (with the molecule residing in the $x z$ plane in the laboratory coordinate frame, see Figure 2). For each value of the inclination angle, a single step 
of a Brownian dynamics simulation (equation 15) was performed. Its duration ( $\Delta t$ in equation 15) was set to $5 \times 10^{-4}$ (in dimensionless units). Resulting translational and angular displacements of the ellipsoid were collected. For each value of the inclination angle, this procedure was repeated $6 \times 10^{6}$ times. Next, all components of the mobility tensor matrix were evaluated based on equation 19 and their values compared with corresponding values calculated using analytical expressions. Nearly perfect agreement evidenced in Figure S2 validates the Brownian dynamics algorithm employed in the current work.
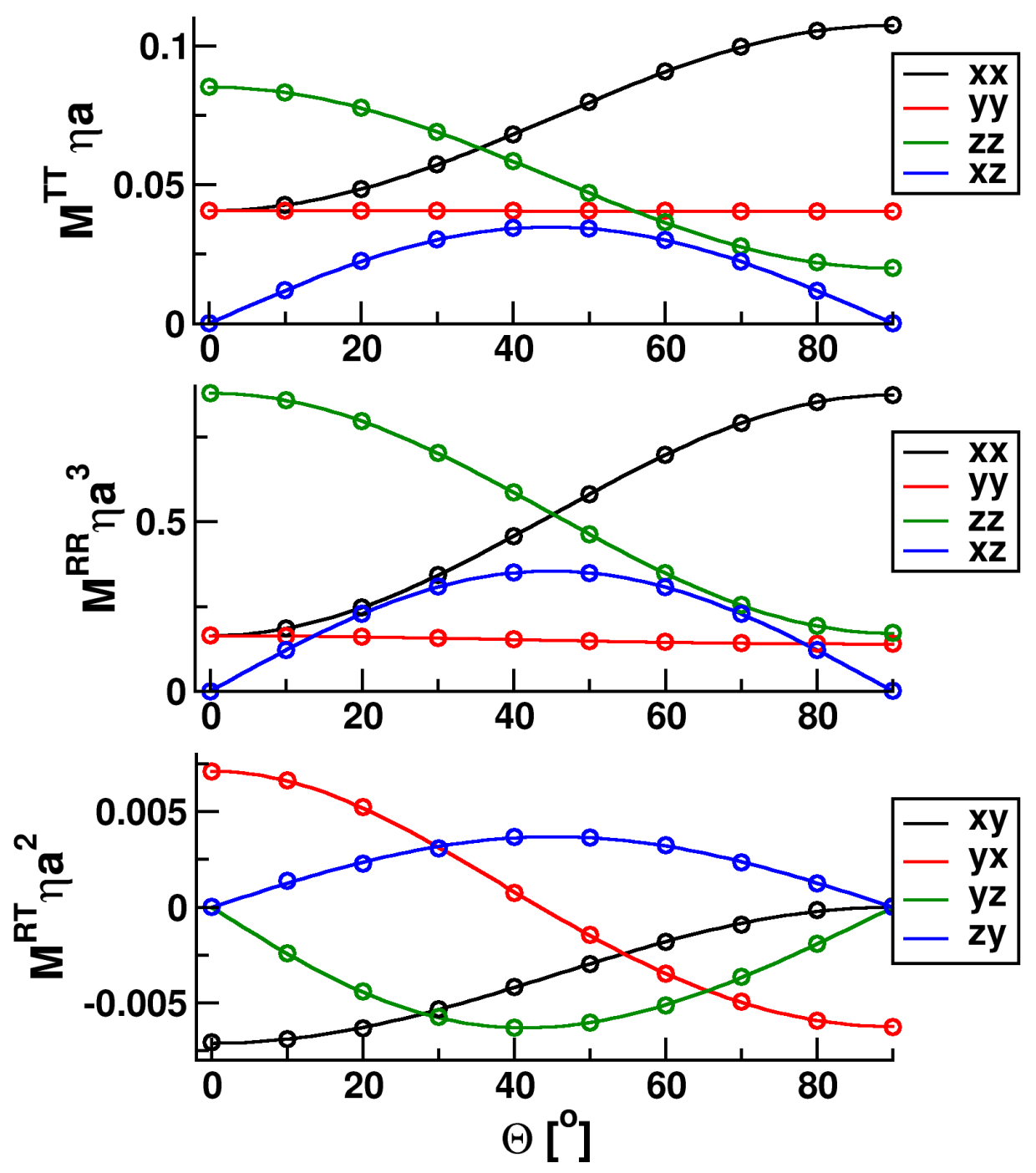

Figure S2: Comparison between various components of the ellipsoid's mobility tensor matrix, computed for different values of the inclination angle $\theta$, from Brownian dynamics simulations (circles, error bars are comparable with symbol sizes) and using analytical expressions given in [1] (continuous lines). Results obtained for an ellipsoid of the axial ratio 4, elevated by $1.125 a$ above the planar surface.

Results of yet another test that we performed to confirm that the propagation algorithm is correct are given in Figure S3. Here we show that, as previously described [3, 4], the inclusion of spatial and orientational divergence terms in equation 15 is necessary to obtain a correct equilibrium distribution of ellipsoid-plane distances. As external forces are absent in 
the studied system, flat distributions were obtained from Brownian dynamics simulations of near-surface diffusion and simulations in an unbounded fluid. Additionally, we also show the distribution obtained from simulations of an ellipsoid diffusing near the plane, in which divergence terms were not included in the equation of ellipsoid's motion, resulting in a falsely increased probability of finding the molecule near the surface.

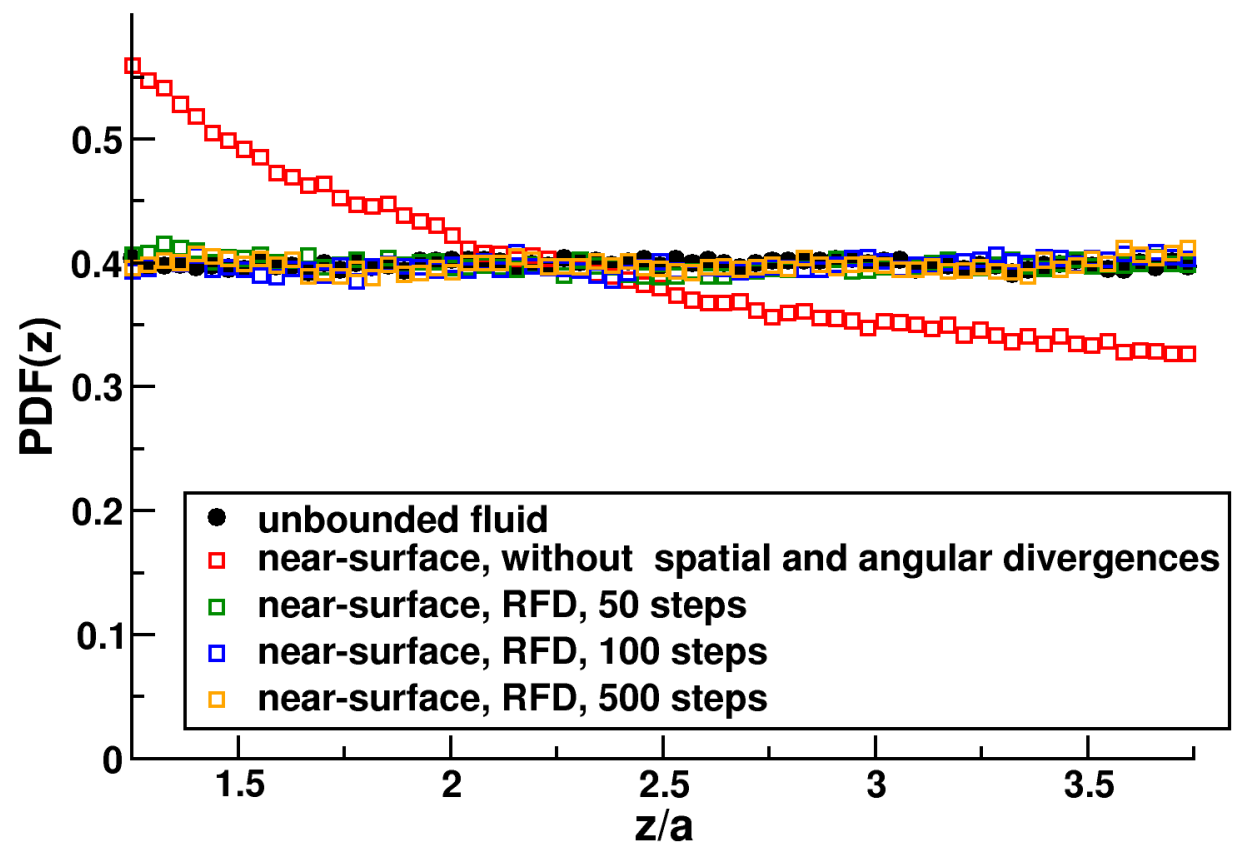

Figure S3: Equilibrium probability distribution functions (PDF) of the ellipsoid-surface distance obtained from Brownian dynamics simulations that either account for (via the random finite difference algorithm (RFD) [4, 5], with a number of random steps indicated in the legend) or neglect the spatial and angular divergences of the mobility matrix. For a comparison, the distribution obtained from Brownian dynamics simulations of an ellipsoid suspended in an unbounded fluid is also shown. Results obtained for an ellipsoid of the axial ratio 4.

Probability distribution functions shown in Figure S3 were computed based on Brownian dynamics trajectories of $2 \times 10^{8}$ steps, during which positions of the ellipsoid were restricted in the domain between $z=a$ and $z=4 a$ (Figure 2). For near-surface simulations the boundary plane was located at $z=0$ (all orientations of the ellipsoid near the surface are allowed). Restriction on ellipsoid's translations in the direction normal to the surface is imposed using an algorithm described previously [6,4], by rejecting Brownian dynamics steps that result in ellipsoid's centre $z$ coordinate outside the predefined interval. Figure S3 serves also to illustrate the performance of the random finite difference method in terms of the number of steps used to evaluate averages in equations 24 and 25 . 


\section{Generation of Non-Gaussian Displacements}

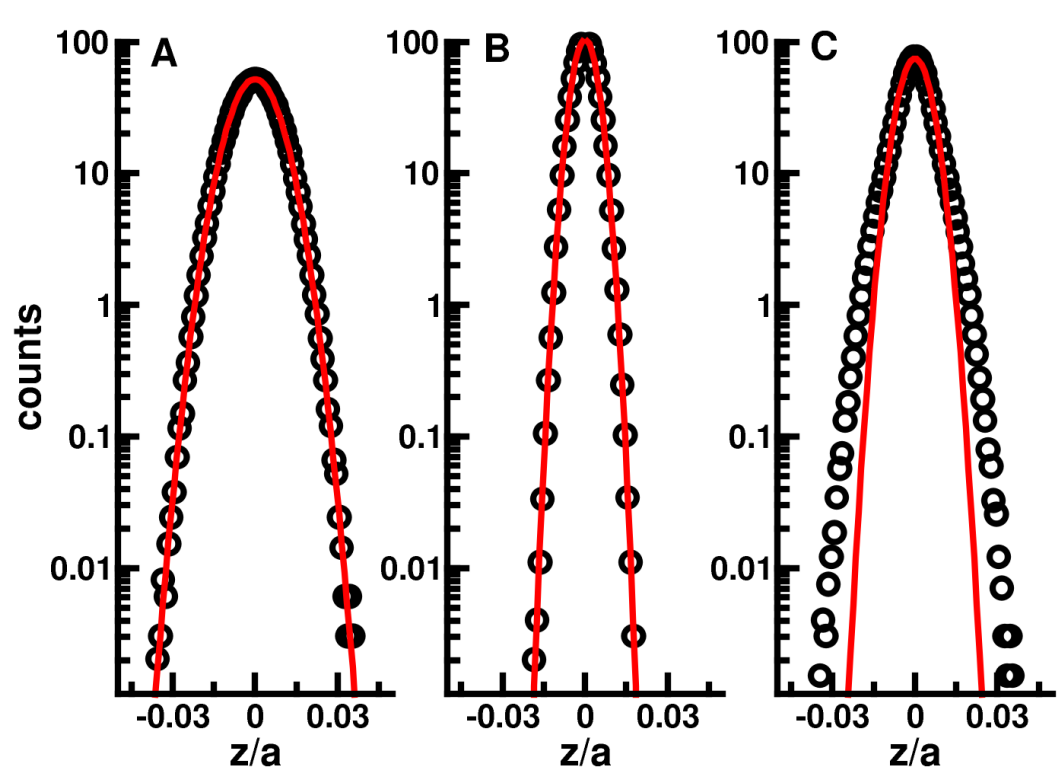

Figure S4: Circles represent distributions of displacements in the $z$ direction of the laboratory coordinate frame, obtained from $10^{6}$ single-step Brownian dynamics simulations of an ellipsoidal protein, of axial ratio 3, suspended near a planar surface, at a fixed elevation of $1.25 a$, for an inclination angle, $\theta$, fixed at a particular value, whereas red continuous lines represent Gaussian fits. A: $\theta=0^{\circ}$; B: $\theta=90^{\circ}$; C: distribution obtained for an ensemble containing displacements collected for $\theta=0^{\circ}$ and $\theta=90^{\circ}$, in equal proportion.

\section{References}

[1] M. Lisicki, B. Cichocki, and E. Wajnryb. Near-wall diffusion tensor of an axisymmetric colloidal particle. J. Chem. Phys., 145:034904, 2016.

[2] S. Kim and S. J. Karrila. Microhydrodynamics. Principles and Selected Applications. Dover Publications, Inc., Mineola, New York, 2005.

[3] A. W. C. Lau and T. C. Lubensky. State-dependent diffusion: Thermodynamic consistency and its path integral formulation. Phys. Rev. E, 76:011123, 2007.

[4] M. De Corato, F. Greco, G. D'Avino, and P. L. Maffettone. Hydrodynamics and Brownian motions of a spheroid near a rigid wall. J. Chem. Phys., 142:194901, 2015.

[5] S. Delong, F. B. Usabiaga, R. Delgado-Buscalioni, B. E. Griffith, and A. Donev. Brownian dynamics without green's functions. J. Chem. Phys., 140:134110, 2014.

[6] H. X. Zhou. Kinetics of diffusion-influenced reactions studied by Brownian dynamics. J. Phys. Chem., 94:8794-8800, 1990. 